\title{
Self-Motility of an Active Particle Induced by Correlations in the Surrounding Solution
}

\author{
Alvaro Domínguez® ${ }^{1,2, *}$ M. N. Popescu $\oplus^{3, \dagger}$ C. M. Rohwer ${ }^{3,4,5, \$}$ and S. Dietrich ${ }^{3,4, \S}$ \\ ${ }^{1}$ Física Teórica, Universidad de Sevilla, Apdo. 1065, 41080 Sevilla, Spain \\ ${ }^{2}$ Instituto Carlos I de Física Teórica y Computacional, 18071 Granada, Spain \\ ${ }^{3}$ Max-Planck-Institut für Intelligente Systeme, Heisenbergstraße 3, 70569 Stuttgart, Germany \\ ${ }^{4} I V$. Institut für Theoretische Physik, Universität Stuttgart, Pfaffenwaldring 57, D-70569 Stuttgart, Germany \\ ${ }^{5}$ Department of Mathematics \& Applied Mathematics, University of Cape Town, 7701 Rondebosch, Cape Town, South Africa
}

(Received 5 June 2020; revised 9 September 2020; accepted 30 October 2020; published 28 December 2020)

\begin{abstract}
Current models of phoretic transport rely on molecular forces creating a "diffuse" particle-fluid interface. We investigate theoretically an alternative mechanism, in which a diffuse interface emerges solely due to a nonvanishing correlation length of the surrounding solution. This mechanism can drive self-motility of a chemically active particle. Numerical estimates indicate that the velocity can reach micrometers per second. The predicted phenomenology includes a bilinear dependence of the velocity on the activity and a possible double velocity reversal upon varying the correlation length.
\end{abstract}

DOI: 10.1103/PhysRevLett.125.268002

Out-of-equilibrium behavior has been a research field of sustained interest, relevant to understanding the emergence of complexity [1-4]. The last decade witnessed a rapidly growing engagement with self-phoretic, chemically active particles as a new paradigm thereof, which exhibits a wealth of phenomena, such as microphase separation [5], self-assembly of superstructures [6], and self-organized patterns of collective motion [7,8]. Additionally, phoresis has proven to be an effective, versatile transport mechanism at the microscale [9-11], leading to a significant resurgence of interest also from a technological perspective.

Following the landmark review by Anderson [12], the phenomenon of phoresis of particles suspended in a fluid is characterized by the presence of a driving thermodynamic field and by a transition region (also called "diffuse interface") around the particle. The forces relevant for the phoretic displacement act within this region: its nonvanishing width gives rise to a response of the fluid medium even though the net sum of the forces and torques vanishes. Thus, the particle is transported by the driving field while the system (particle plus fluid) is mechanically isolated, which sets this effect fundamentally apart from that of transport by an external force. The thermodynamic driving field usually comprises an externally imposed gradient in one of the state parameters (such as composition, temperature, etc.) of the fluid, or of an electric field in the case of a charged particle in an electrolyte. The alternative case, in which the particle

Published by the American Physical Society under the terms of the Creative Commons Attribution 4.0 International license. Further distribution of this work must maintain attribution to the author(s) and the published article's title, journal citation, and DOI. itself generates the driving field- $\mathrm{a}$ scenario also envisioned by Anderson [12] — has recently received much attention under the generic name of "self-phoresis" [13-16]. Experimentally, self-propelled particles have been realized most often in the form of so-called Janus particles. In a typical scenario, such particles are immersed in a multicomponent fluid solution and promote catalytic reactions which vary across their surfaces, leading to selfelectrophoresis $[17,18]$ or self-chemiophoresis $[5,13,19-21]$. Alternatively, the particles behave as heat sources, leading to self-thermophoresis through a single-component fluid [22] or self-chemiophoresis via demixing of a critical binary liquid mixture [23].

A common ingredient in the models which have so far addressed phoretic [12] and self-phoretic [15,16,22,24-27] phenomena is a direct physical interaction between the particle and the molecules of the surrounding fluid [28]. The corresponding nonvanishing interaction range provides the aforementioned diffuse interfacial region, where the internal forces and torques act and induce motion. Here, we propose an alternative transport mechanism which does not require this direct, extended interaction: instead, the interfacial region is diffuse because the fluid exhibits a nonvanishing correlation length. Although the phenomenology driven by this mechanism could also be called "phoresis" (in accordance with the definition proposed by Anderson [12]), we coin the notion of "correlation-induced transport" in order to distinguish it from the usual one, which necessarily invokes a direct, spatially extended interaction.

Here, we focus on the example of chemiophoresis, for which physically insightful analytical expressions can be derived, in order to illustrate the correlation-induced transport. However, the conceptual framework discussed below is expected to exhibit a very general applicability for other 
types of phoresis-albeit significantly more technically involved. For clarity and simplicity, we consider a rigid, spherical particle (radius $R$ ), immersed in a solution consisting of a solvent plus a single solute species (called "the chemical" in the following). The particle is active, i.e., it is a source or sink of the chemical. The two main approximations are a spatially and temporally constant temperature and mass density of the solution, and a slow motion of the particle [29]. Consequently, the state of the solution can be characterized [1] by the instantaneous stationary profiles of, e.g., the mass density of the chemical or, equivalently, of its number density $n(\mathbf{r})$ valid for small Péclet numbers, and of the velocity field $\mathbf{u}(\mathbf{r})$ of the solution valid for small Reynolds and Mach numbers. Thus, $n(\mathbf{r})$ is determined by the constraint of mass conservation for the chemical,

$$
\frac{\partial n}{\partial t}=-\nabla \cdot \mathbf{j}_{\text {diff }}=0, \quad \mathbf{j}_{\text {diff }}=\Gamma \mathbf{f} .
$$

Here, $\Gamma$ is the mobility of the chemical within the solution, while $\mathbf{f}$ is the body force density acting on the chemical and which drives its diffusion. Likewise, $\mathbf{u}(\mathbf{r})$ follows from the balance between this body force and the fluid stresses, as expressed by the Stokes equation for incompressible flow,

$$
\nabla \cdot \Pi+\mathbf{f}=0, \quad \nabla \cdot \mathbf{u}=0,
$$

in terms of the stress tensor $\Pi=\eta\left[\nabla \mathbf{u}+(\nabla \mathbf{u})^{\dagger}\right]-\mid p$ ( $\eta$ is the viscosity of the solution, $p$ the pressure field enforcing incompressibility, and I the identity tensor). Complementarily to the slow particle dynamics, one assumes local equilibrium for the chemical [12], so that

$$
\mathbf{f}(\mathbf{r})=-n(\mathbf{r}) \nabla \mu(\mathbf{r}),
$$

in terms of its chemical potential $\mu$. The latter is modeled by means of a free energy functional [40] [we take spherical coordinates $(r, \theta, \varphi)$ with the origin at the center of the particle; $d \mathcal{V}$ denotes the volume element]

$$
\mathcal{H}[n]=\int_{|\mathbf{r}|>R} d \mathcal{V}\left[h(n)+\frac{1}{2} \lambda^{2}|\nabla n|^{2}+n W(\mathbf{r})\right],
$$

so that

$$
\mu(\mathbf{r})=\frac{\delta \mathcal{H}[n]}{\delta n(\mathbf{r})}=h^{\prime}(n(\mathbf{r}))-\lambda^{2} \nabla^{2} n(\mathbf{r})+W(\mathbf{r}) .
$$

Here, $h(n)$ is a local free energy density, and $W(\mathbf{r})$ is a potential energy (which has a nonzero and finite range) generated by the particle [41]. In Eq. (4), the term $\propto \lambda^{2}$ accounts for the range of the molecular interactions between the dissolved molecules of the chemical [42]. The relevant control parameters of this model for the chemical are $\lambda$ and the temperature (implicit in the definition of $\mathcal{H}$ ). However, it turns out [see cf. Eq. (12)] that the results depend only on $\lambda$ and the correlation length $\xi:=\lambda / \sqrt{h^{\prime \prime}\left(n_{0}\right)}$ for a reference density $n_{0}$ [43], without the need to specify the dependence on temperature.

Finally, one has to specify boundary conditions: at infinity, a homogeneous equilibrium state is recovered, i.e., a vanishing velocity of the fluid solution and a constant chemical potential due to a reservoir,

$$
\mathbf{u}(\mathbf{r}) \rightarrow 0, \quad \mu(\mathbf{r}) \rightarrow \mu_{0}, \quad \text { as }|\mathbf{r}| \rightarrow \infty,
$$

thereby also fixing the density at a value $n_{0}$ such that $h^{\prime}\left(n_{0}\right)=\mu_{0}$. On the surface of the particle, we impose a no-slip boundary condition in terms of the translational $(\mathbf{V})$ and angular $(\boldsymbol{\Omega})$ velocities of the particle,

$$
\mathbf{u}(\mathbf{r})=\mathbf{V}+\mathbf{\Omega} \times \mathbf{r}, \quad \text { at }|\mathbf{r}|=R,
$$

the boundary condition for the density, which follows from the surface term of the variation of the free energy functional [44],

$$
\lambda^{2} \mathbf{e}_{r} \cdot \nabla n(\mathbf{r})=0, \quad \text { at }|\mathbf{r}|=R,
$$

and a prescribed current of the chemical, modeling the activity of the particle as a source of the chemical:

$$
\mathbf{e}_{r} \cdot \mathbf{j}_{\text {diff }}(\mathbf{r})=q \mathbb{A}(\theta, \varphi), \quad \text { at }|\mathbf{r}|=R .
$$

The positive constant $q$ represents the production rate of the chemical per area of the particle, and $\mathbb{A}(\theta, \varphi)$ is the dimensionless activity, which can vary along the surface of the particle.

Equations (1)-(9), together with the condition that the particle does not experience any external force or torque, constitute a complete description for obtaining the velocities $\mathbf{V}$ and $\boldsymbol{\Omega}$ of the particle for a prescribed activity function $\mathbb{A}$. If $q=0$, the equilibrium state $\mu(\mathbf{r})=\mu_{0}$ is a solution of the equations, which implies $\mathbf{V}=0$ and $\boldsymbol{\Omega}=0$. Thus, particle transport requires the nonequilibrium imbalance introduced by the chemical activity. We address two important aspects for solving this model. First, mechanical isolation of the whole system "particle + solution" holds because, according to Eq. (2), the medium transmitting forces from the particle to infinity is in local mechanical balance [29]. Second, according to Eq. (2), the incompressibility constraint implies that the motion is driven by the spatially extended field $\nabla \times \mathbf{f}$, which (because of mechanical isolation) is the only source of flow vorticity [45]. Combining Eqs. (3) and (5), one obtains

$$
\begin{aligned}
\nabla \times \mathbf{f} & =\nabla \mu \times \nabla n \\
& =\nabla W \times \nabla n-\lambda^{2} \nabla \times\{\nabla \cdot[(\nabla n)(\nabla n)]\} .
\end{aligned}
$$

Therefore, the transport, i.e., $\nabla \times \mathbf{f} \neq 0$, requires either an explicit interaction between the particle and the chemical, 
i.e., $W \neq 0$, or a deviation of the solution from ideality, i.e., $\lambda \neq 0$, which is associated with a nonvanishing correlation length in the solution. To the best of our knowledge, all previous studies of phoresis have analyzed and identified the case $W \neq 0$ as the primary origin of particle transport. Here, we address the opposite case $(W=0, \lambda \neq 0)$, for which the model [Eqs. (1)-(9)] implies that the spatially homogeneous equilibrium state $\left[n(\mathbf{r})=n_{0}, \mathbf{u}(\mathbf{r})=0\right]$ would not be perturbed by the presence of a passive particle, i.e., $q=0$. This case isolates the effect of correlations and already provides distinctive observable predictions.

A complete analytical solution for the correlationinduced transport velocity can be obtained if the deviations from homogeneity are small, i.e., for small values (weak activity) of the Damköhler number $\mathrm{Da}=q R \beta /\left(n_{0} \Gamma\right)$, where $\beta$ is the inverse thermal energy and $\Gamma / \beta$ is the diffusivity of the chemical: the dimensionless ratio $\mathrm{Da}$ quantifies the activity-induced "chemical crowding" near the particle against the opposing effect of diffusion. In this case, the spatial variation of $\eta$ and $\Gamma$ (via a possible dependence on the density $n$ ) can be neglected. Consequently, the "diffusion problem" for the density profile $n(\mathbf{r})$, posed by Eqs. (1), (3), (5), (6), (8), and (9), is decoupled from the velocity field and can be solved first on its own. This can be carried out analytically, after linearizing in the small quantity $n(\mathbf{r})-n_{0}$. Subsequently, one solves the "hydrodynamical problem," stated by Eqs. (2), (6), and (7), with the body force in Eq. (3) determined by the profile $n(\mathbf{r})$ obtained in the first step. Conveniently, the Lorentz reciprocal theorem allows one to sidestep the full solution of the hydrodynamical problem and to obtain expressions for $\mathbf{V}$ and $\boldsymbol{\Omega}$ directly in terms of $n(\mathbf{r})$. Details of this procedure are collected in the Supplemental Material [29]. By expanding the activity in terms of spherical harmonics,

$$
\mathbb{A}(\theta, \varphi)=\sum_{\ell=0}^{\infty} \sum_{m=-\ell}^{\ell} a_{\ell m} Y_{\ell m}(\theta, \varphi)
$$

one obtains a compact expression for the correlationinduced transport velocity:

$$
\begin{aligned}
\mathbf{V}= & V_{0} \sum_{\ell m} \sum_{\ell^{\prime} m^{\prime}} a_{\ell m} a_{\ell^{\prime} m^{\prime}} \\
& \times\left[g_{\ell \ell^{\prime}}^{\perp}(\xi / R) \mathbf{G}_{\ell m ; \ell^{\prime} m^{\prime}}^{\perp}+g_{\ell \ell^{\prime}}^{\|}(\xi / R) \mathbf{G}_{\ell m ; \ell^{\prime} m^{\prime}}^{\|}\right]
\end{aligned}
$$

with the velocity scale $V_{0}:=\mathrm{Da}^{2} R^{3} /\left(6 \pi \eta \beta^{2} \lambda^{2}\right)$. In the Supplemental Material we provide the rather lengthy expressions for the purely geometrical factors $\mathbf{G}$, which depend only on the mathematical properties of the spherical harmonics, and for the dimensionless coefficients $g$, which fully incorporate the dependence on the correlation length $\xi$ brought about by the physical model [46].
The superscripts $\perp$ and $\|$ denote the contributions by the radial and tangential components of the body force $\mathbf{f}$, respectively. The angular velocity $\boldsymbol{\Omega}$ turns out to vanish identically (see below).

Several general conclusions can be extracted already from this expression. Since Eq. (12) is bilinear in the activity, the velocity is invariant under the transformation $a_{\ell m} \rightarrow-a_{\ell m}, \forall \ell, m$. This means that the direction of translation is insensitive to whether the particle acts as a source or a sink of the exchanged chemical. As shown by Eq. (10), a nonvanishing velocity requires a deviation from equilibrium $(\nabla \mu \neq 0)$, as well as a deviation from homogeneity $(\nabla n \neq 0)$. In the model of correlation-induced transport, both requirements are enforced by the activity, which explains the bilinear dependence. This is at variance with the case of standard phoresis, which gives rise to a velocity linear in the activity [29] because $W \neq 0$ usually suffices to bring about a relevant inhomogeneity. Another consequence of the symmetric form of the term $\propto \lambda^{2}$ in Eq. (10) is the absence of chirality, so that $\boldsymbol{\Omega}=0$ [47]. In the standard model, on the contrary, the relative orientation of $\nabla n$ and $\nabla W$ will provide, in general, a preferred direction and sense of rotation, thus inducing a nonvanishing angular velocity.

The geometrical factors $\mathbf{G}_{\ell m ; \ell^{\prime} m^{\prime}}^{\perp}$ and $\mathbf{G}_{\ell m ; \ell^{\prime} m^{\prime}}^{\|}$can be expressed in terms of the Wigner $3 j$ symbols [29], which induce "selection rules": these factors will vanish unless $\left|\ell-\ell^{\prime}\right|=1$ and $\left|m+m^{\prime}\right| \leq 1$. Therefore, the sum in Eq. (12) explicitly couples only pairs of "neighboring multipoles." This result captures a major difference as compared to standard phoresis. For instance, an activity pattern lacking a dipole in Eq. (11) generically yields $\mathbf{V} \neq 0$, while the same pattern of activity, together with a spherically symmetric potential $W(r)$, does not yield phoresis in the standard model [48].

Another interesting feature of the transport velocity is revealed by considering the limit $\xi \ll R$ : the deviations from homogeneity are then localized within a thin layer (of thickness $\propto \xi$ ) at the surface of the particle. In this case, the velocity $\mathbf{V}$ can be expressed as an integral over the surface of the particle [29]:

$$
\mathbf{V} \approx-\frac{1}{4 \pi} \int_{0}^{\pi} d \theta \sin \theta \int_{0}^{2 \pi} d \varphi \mathbf{v}_{\text {slip }}(\theta, \varphi) .
$$

The integrand can be interpreted as a slip velocity for the hydrodynamical problem [12], which is proportional to the tangential component of the density gradient,

$$
\mathbf{v}_{\text {slip }}(\mathbf{r}):=\mathcal{L}(\mathbf{r}) \nabla_{\|} n(\mathbf{r}), \quad \text { at }|\mathbf{r}|=R,
$$

with a local coefficient of phoretic mobility,

$$
\mathcal{L}(\theta, \varphi):=\frac{\mathrm{Da}^{3}}{\eta \beta R} \mathbb{A}(\theta, \varphi) .
$$




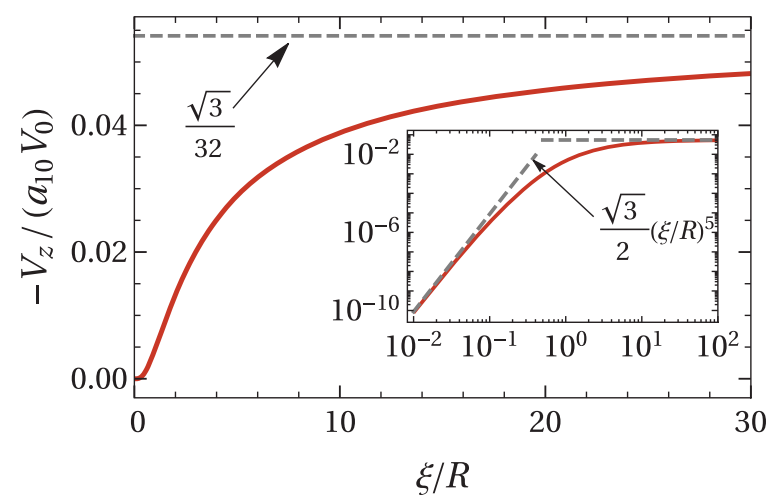

FIG. 1. Dimensionless $z$ component of the correlation-induced transport velocity as a function of the correlation length $\xi$ for the simplest case with polar symmetry (see the main text for details). The inset shows the same data on log scales; the dashed lines indicate the asymptotic behaviors as $\xi \rightarrow 0$ and $\infty$, respectively.

Because of the explicit dependence of $\mathcal{L}$ on the activity (via $\mathrm{Da})$, the present mechanism of self-propulsion cannot be interpreted as "passive" transport in an external (albeit selfinduced) gradient, which is another significant deviation from standard phoresis [49]. Finally, we note that $V \sim \xi^{5}$ if $\xi \ll R$ because the tangential gradient vanishes $\propto \xi^{2}$ [29]. In the opposite limit $\xi \gg R$, the velocity given by Eq. (12) approaches a finite value [51], which depends only on $V_{0}$ and $a_{\ell m}$.

As an illustration [29], we study the simplest activity distribution with polar symmetry by retaining only monopolar and dipolar contributions: $a_{00}=1, a_{1 m}=a_{10} \delta_{m, 0}$, and $a_{\ell \geq 2}=0$ in Eq. (11). For this case, Eq. (12) renders $\mathbf{V}=\mathbf{e}_{z} V_{z}, V_{z}=a_{10} V_{0} f_{1}(\xi / R)$, with the function $f_{1}(\xi / R)$ shown in Fig. 1. The velocity depends linearly on the dipole strength $a_{10}$ and vanishes in the spherically symmetric limit $a_{10} \rightarrow 0$. The asymptotic behaviors for $\xi \ll R$ or $\xi \gg R$ are given by the general results just discussed. If an additional quadrupolar activity contribution $a_{20} \neq 0$ is included, Eq. (12) gives $V_{z}=a_{10} V_{0}\left[f_{1}(\xi / R)+a_{20} f_{2}(\xi / R)\right]$, where $f_{1}$ and $f_{2}$ are monotonic functions of $\xi$ with opposite signs. Strikingly, this implies that the sign of $V_{z}$ can be altered by merely changing the correlation length $\xi$ (e.g., through temperature), while keeping the particle properties (i.e., the coefficients $a_{\ell m}$ ) fixed. This rich behavior is illustrated in Fig. 2. For certain values of $a_{20}$, such as $a_{20}=7$, one indeed observes a reentrant regime where $V_{z}$ is negative at very small and very large $\xi$, but positive in an intermediate range of correlation lengths.

These results allow one to estimate the magnitude of the velocity under realistic conditions. From Fig. 1, upon taking $a_{10}=1$, one obtains $V_{z} \approx V_{0}(\xi / R)^{5}$ in the regime $\xi / R<1$. In order to estimate the velocity scale $V_{0}$ [see below Eq. (12)], we approximate the local free energy of the chemical by the ideal gas expression $\beta h(n)=n(\ln n-1)$, so that $\xi^{2}=\beta n_{0} \lambda^{2}$ and $V_{0}=(\mathrm{Da} R / \xi)^{2} n_{0} R /(6 \pi \eta \beta)$. With the typical values

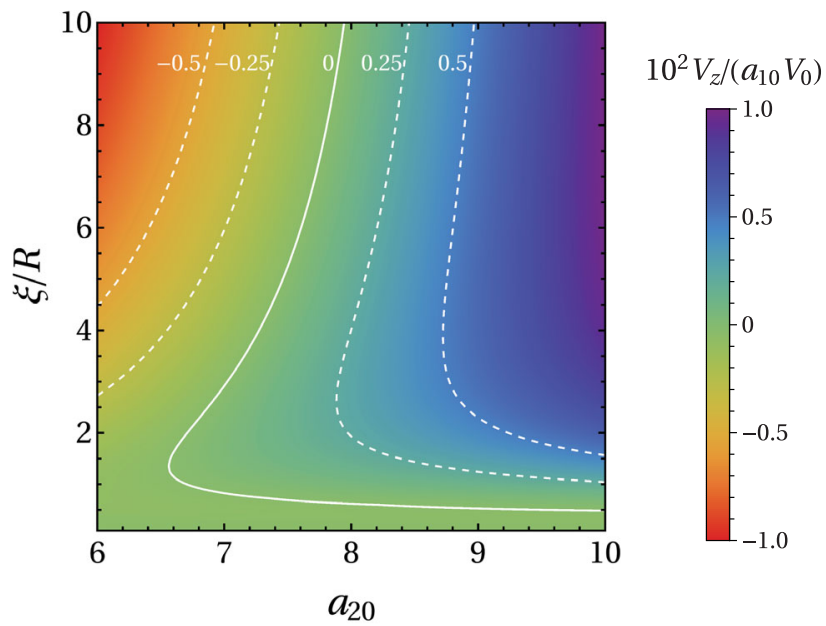

FIG. 2. Dimensionless $z$ component of the correlation-induced transport velocity as a function of the correlation length $\xi$ and of the quadrupolar activity parameter $a_{20}$ (see the main text for details). The white curves are contour lines.

$R=1 \mu \mathrm{m}, \quad n_{0}=1 \mathrm{mM}, \quad \eta=10^{-3}$ Pas (viscosity of water), and $\mathrm{Da}=10^{-1}$ (as for, e.g., the Pt catalyzed decomposition of hydrogen peroxide reported in Refs. [17,26]), one obtains $V_{z} \sim(10 \xi / R)^{3} \mu \mathrm{m} / \mathrm{s}$ at room temperature. For $\xi / R=10^{-1}$, the predicted velocity would be easily measurable; actually, the strong dependence on $\xi / R$ provides a broad range of variation covering the values reported from experiments for distinct types of systems [19-21,52,53].

In summary, we have studied a mechanism of phoresis driven by correlations in the solution. Significant differences with respect to the standard mechanism of phoresis become evident. (i) The correlation-induced selfpropulsion is bilinear in the activity, so that for the same activity pattern the two mechanisms predict distinct observable velocities. (ii) There is no self-rotation, regardless of the activity pattern. (iii) The correlation-induced phoresis cannot be understood as passive phoresis in an external (albeit self-induced) driving field. Already for simple activity patterns, the self-propulsion velocity exhibits remarkable features, including reentrant changes of sign obtained upon varying the correlation length (see Fig. 2). Numerical estimates for realistic conditions give values comparable to those observed experimentally, which are usually interpreted within the framework of the standard model of phoresis; correlation-induced phoresis provides an additional, plausible mechanism for addressing these observations.

Thus, our formalism opens the door to studying the role which correlations play in phoretic phenomena for a broad class of systems and geometries. In the physically relevant situation in which $\xi \neq 0$ and $W \neq 0$, both correlationinduced transport and common phoresis would occur, including a coupling between correlations within the fluid and the inhomogeneities induced by $W(\mathbf{r})$. An extension of 
our analytical model could address their relative importance [50]. In this regard, the present study constitutes, inter alia, an important addition to the theoretical machinery which is relevant for sorting and interpreting recent results pertaining to self-propulsion due to demixing in a binary liquid mixture [its order parameter playing the role of $n(\mathbf{r})]$. For example, this encompasses the numerical analysis in Ref. [25], which incorporates nonlinear couplings (due to a nonvanishing Péclet number) in order to explain the emergence of self-motility, and the experimental observation of velocity reversals for certain self-propelled particles [54] following a change of illumination intensity (which changes the temperature distribution in the fluid and, correspondingly, the correlation length).

To conclude, we have identified and characterized a novel mechanism for self-phoresis of an active particle, which features potentially observable differences in comparison with the mechanism considered so far in the literature, and which can be controlled, e.g., via varying the temperature of the bath.

A. D. acknowledges support by the Spanish Government through Grant No. FIS2017-87117-P (partially financed by FEDER funds).

*dominguez@us.es

†popescu@is.mpg.de

*crohwer@is.mpg.de

§ietrich@is.mpg.de

[1] S. R. de Groot and P. Mazur, Non-Equilibrium Thermodynamics (Dover, New York, 1984).

[2] F. Sagués, J. M. Sancho, and J. García-Ojalvo, Spatiotemporal order out of noise, Rev. Mod. Phys. 79, 829 (2007).

[3] E. Branscomb, T. Biancalani, N. Goldenfeld, and M. Russell, Escapement mechanisms and the conversion of disequilibria; the engines of creation, Phys. Rep. 677, 1 (2017).

[4] S. I. Walker, Origins of life: A problem for physics, a key issues review, Rep. Prog. Phys. 80, 092601 (2017).

[5] J. Palacci, S. Sacanna, A. P. Steinberg, D. J. Pine, and P. M. Chaikin, Living crystals of light-activated colloidal surfers, Science 339, 936 (2013).

[6] A. Aubret, M. Youssef, S. Sacanna, and J. Palacci, Targeted assembly and synchronization of self-spinning microgears, Nat. Phys. 14, 1114 (2018).

[7] F. A. Lavergne, H. Wendehenne, T. Bäuerle, and C. Bechinger, Group formation and cohesion of active particles with visual perception-dependent motility, Science 364, 70 (2019).

[8] D. P. Singh, A. Domínguez, U. Choudhury, S. N. Kottapalli, M. N. Popescu, S. Dietrich, and P. Fischer, Interfacemediated spontaneous symmetry breaking and mutual communication between drops containing chemically active particles, Nat. Commun. 11, 2210 (2020).

[9] S. Marbach and L. Bocquet, Osmosis, from molecular insights to large-scale applications, Chem. Soc. Rev. 48, 3102 (2019).
[10] S. Battat, J. T. Ault, S. Shin, S. Khodaparast, and H. A. Stone, Particle entrainment in dead-end pores by diffusiophoresis, Soft Matter 15, 3879 (2019).

[11] T. Tsuji, Y. Sasai, and S. Kawano, Thermophoretic Manipulation of Micro- and Nanoparticle Flow through a Sudden Contraction in a Microchannel with Near-Infrared Laser Irradiation, Phys. Rev. Applied 10, 044005 (2018).

[12] J.L. Anderson, Colloid transport by interfacial forces, Annu. Rev. Fluid Mech. 21, 61 (1989).

[13] R. Golestanian, T. B. Liverpool, and A Ajdari, Propulsion of a Molecular Machine by Asymmetric Distribution of Reaction Products, Phys. Rev. Lett. 94, 220801 (2005).

[14] G. Ruckner and R. Kapral, Chemically Powered Nanodimers, Phys. Rev. Lett. 98, 150603 (2007).

[15] R. Golestanian, T. B. Liverpool, and A Ajdari, Designing phoretic micro- and nano-swimmers, New J. Phys. 9, 126 (2007).

[16] J. L. Moran and J. D. Posner, Phoretic self-propulsion, Annu. Rev. Fluid Mech. 49, 511 (2017).

[17] W. F. Paxton, K. C. Kistler, C. C. Olmeda, A. Sen, S. K. St. Angelo, Y. Y. Cao, T. E. Mallouk, P. E. Lammert, and V. H. Crespi, Catalytic nanomotors: Autonomous movement of striped nanorods, J. Am. Chem. Soc. 126, 13424 (2004).

[18] S. Fournier-Bidoz, A. C. Arsenault, I. Manners, and G. A. Ozin, Synthetic self-propelled nanorotors, Chem. Commun. 441 (2005).

[19] A. Brown and W. Poon, Ionic effects in self-propelled Ptcoated Janus swimmers, Soft Matter 10, 4016 (2014).

[20] J. Simmchen, J. Katuri, W. E. Uspal, M. N. Popescu, M. Tasinkevych, and S. Sánchez, Topographical pathways guide chemical microswimmers, Nat. Commun. 7, 10598 (2016).

[21] D. P. Singh, U. Choudhury, P. Fischer, and A. G. Mark, Non-equilibrium assembly of light-activated colloidal mixtures, Adv. Mater. 29, 1701328 (2017).

[22] K. Kroy, D. Chakraborty, and F. Cichos, Hot microswimmers, Eur. Phys. J. Special Topics 225, 2207 (2016).

[23] G. Volpe, I. Buttinoni, D. Vogt, H.-J. Kümmerer, and C. Bechinger, Microswimmers in patterned environments, Soft Matter 7, 8810 (2011).

[24] A. Würger, Self-Diffusiophoresis of Janus Particles in Near-Critical Mixtures, Phys. Rev. Lett. 115, 188304 (2015).

[25] S. Samin and R. van Roij, Self-Propulsion Mechanism of Active Janus Particles in Near-Critical Binary Mixtures, Phys. Rev. Lett. 115, 188305 (2015).

[26] A. T. Brown, W. C. K. Poon, C. Holm, and J. de Graaf, Ionic screening and dissociation are crucial for understanding chemical self-propulsion in polar solvents, Soft Matter 13, 1200 (2017).

[27] Y. Ibrahim, R. Golestanian, and T. B. Liverpool, Multiple phoretic mechanisms in the self-propulsion of a Pt-insulator Janus swimmer, J. Fluid Mech. 828, 318 (2017).

[28] The paradigmatic setups one has considered involve either electrostatic forces or dispersion forces between the particle and the components of the fluid solution [16].

[29] See Supplemental Material at http://link.aps.org/ supplemental/10.1103/PhysRevLett.125.268002 for full details, which includes Refs. [30-39]. 
[30] J. Happel and H. Brenner, Low Reynolds Number Hydrodynamics (Prentice-Hall, Englewood Cliffs, NJ, 1965).

[31] Handbook of Mathematical Functions, edited by M. Abramowitz and I. R. Stegun (Dover, New York, 1972).

[32] I. S. Gradshteyn and I. M. Ryzhik, Table of Integrals, Series, and Products, 5th ed., edited by A. Jeffrey (Academic Press, London, 1994).

[33] H. A. Lorentz, Eene algemeene stelling omtrent de beweging eener vloeistof met wrijving en eenige daaruit afgeleide gevolgen, Z. K. Akad. Wet. Amsterdam 5, 168 (1896).

[34] H. K. Kuiken, H. A. Lorentz: A general theorem on the motion of a fluid with friction and a few results derived from it (translated from Dutch by H. K. Kuiken), J. Eng. Math. 30, 19 (1996).

[35] M. Teubner, Motion of charged colloidal particles, J. Chem. Phys. 76, 5564 (1982).

[36] S. Kim and S. J. Karrila, Microhydrodynamics: Principles and Selected Applications (Butterworth-Heinemann, New York, 1991).

[37] A. Messiah, Quantum Mechanics (Dover, New York, 1999).

[38] C. M. Bender and S. A. Orszag, Advanced Mathematical Methods for Scientists and Engineers (McGraw-Hill, New York, 1978).

[39] B. V. Derjaguin, G. P. Sidorenkov, E. A. Zubashchenkov, and E. V. Kiseleva, Kinetic phenomena in boundary films of liquids, Kolloidn. Zh. 9, 335 (1947).

[40] D. M. Anderson, G. B. McFadden, and A. A. Wheeler, Diffuse-interface methods in fluid mechanics, Annu. Rev. Fluid Mech. 30, 139 (1998).

[41] For an incompressible solution, $W$ is actually the difference in interaction of the particle with a molecule of solute and with a molecule of solvent, respectively [1].

[42] More precisely, $\lambda$ can be directly related to the second moment of the attractive (e.g., van der Waals) part of the pair potential between the molecules of the chemical.

[43] The free energy in Eq. (4), with $W=0$ and linearized in $\delta n=n-n_{0}$, provides the equilibrium correlation function $\langle\delta n(\mathbf{r}) \delta n(\mathbf{0})\rangle \sim r^{-1} e^{-r / \xi}$.
[44] In the limit $\lambda \rightarrow 0$, this boundary condition becomes actually irrelevant.

[45] The force can be decomposed into potential (i.e., irrotational) and solenoidal (i.e., divergence-free) components, $\mathbf{f}=\mathbf{f}_{\text {pot }}+\mathbf{f}_{\text {sol }}$. The potential component, $\mathbf{f}_{\text {pot }}=-\nabla \mathcal{P}$, can be absorbed by the (auxiliary) hydrodynamic pressure field, $p \mapsto p+\mathcal{P}$, that enforces incompressibility; see also Eqs. (III.19) and (III.22) in Ref. [29].

[46] See Eqs. (III.39)-(III.42) in Ref. [29].

[47] See the discussion of Eq. (IV.16) in Ref. [29].

[48] See Ref. [15]. There is also the complementary case: A purely dipolar pattern (i.e., the simplest model of a Janus particle with no net production) renders $\mathbf{V}=0$ [29], but in the standard model it yields a non-vanishing phoresis even for a spherically symmetric potential.

[49] Correlation-induced phoresis is also predicted for a passive particle in an external density gradient, yielding a transport velocity which is at least cubic in this gradient [50]. This might explain why it has not received attention, being overshadowed by the mechanism based on an extended interaction.

[50] A. Domínguez et al. (to be published).

[51] This conclusion is not trivial because the density field $n(\mathbf{r})$ does require a finite $\xi$ in order to satisfy the boundary condition $n \rightarrow n_{0}$ at infinity [29].

[52] S. Ebbens, M.-H. Tu, J. R. Howse, and R. Golestanian, Size dependence of the propulsion velocity for catalytic Janus-sphere swimmers, Phys. Rev. E 85, 020401(R) (2012).

[53] S. Das, Z. Jalilvand, M. N. Popescu, W. E. Uspal, S. Dietrich, and I. Kretzschmar, Floor- or ceiling-sliding for chemically active, gyrotactic, sedimenting Janus particles, Langmuir 36, 7133 (2020).

[54] J. R. Gomez-Solano, S. Samin, C. Lozano, P. RuedasBatuecas, R. van Roij, and C. Bechinger, Tuning the motility and directionality of self-propelled colloids, Sci. Rep. 7, 14891 (2017). 